\title{
Use of GIS mapping in the COVID 19 outbreak management
}

Sampath Indika Kumara MK, Pathiraja PMRBI, Ranasinghe RASU, Vipula Shantha NAR.

\begin{abstract}
COVID 19 pandemic has exposed gaps in outbreak management in different parts of the world. Evidence based decisions are compelled by health authorities. Even though Geographical Information System (GIS) has been identified as a technology to support different avenues of public health management, it has been rarely utilized at regional level outbreak management. COVID-19 case maps for high-risk Medical Officer of Health $(\mathrm{MOH})$ areas at Grama Niladari Division (GND) level were compiled using freely available GIS software. These maps were used in different scenarios of regional level outbreak management and also helped other nonhealth stakeholders involved in COVID 19 prevention activities.
\end{abstract}

Key word: COVID-19, GIS, Case Mapping.

\section{Introduction}

Geographic Information Systems (GIS) and spatial mapping act as new tools to fight complex pandemics. The use of GIS mapping in an outbreak yields several important results for prevention of further spread, identification of hot spots, resource allocation, future trend projections. The challenges caused by COVID 19 prompted health officials to use GIS mapping. The use of maps can be at many levels. It has been initially used for spatial identification of the spread of the disease and forecasting future outbreaks(1). Several developing countries have practiced GIS based analysis in the COVID 19 outbreak situation in terms of understanding the real scenario. In Australia GPS mapping was used to describe the geographic distribution variability of COVID 19 in reference to health care services availability(2). In South Africa mapping was used to outline the COVID 19 risk zones (3). Maps have been found to be effective in identifying and communicating risk(4). But use of GIS mapping in the field is still in infancy stages in Developing countries like Sri Lanka due to inadequacy GIS professionals and the resources. COVID 19 has become a global pandemic in the recent years with the first few cases reporting in 31/12/2019(5). Since then up to this date, there has been $121,817,583$ COVID 19 cases and 2692699 deaths due to COVID 19 worldwide(6).

First case of COVID 19 in Sri Lanka was reported on 10/03/2020(7). First COVID 19 case in Kurunegala was reported in $13 / 03 / 2020$. Since then 4,556 COVID patients and 66 deaths due to COVID 19 have been reported in North Western Province (NWP)(8). Our objective in this study was to utilize GIS mapping in the COVID 19 outbreak management in local setting.

\section{Design and Methods}

Map is a visual output generated from various data sources for the decision on geographically bounder instances. By nature of the Map, it is easy to understand the real situation in terms of geographical means rather than other classical analysis tools.

In the situation of COVID 19 pandemic, it is most important to obtain the real locational information and recent movement of COVID 19 positive cases to reduce the spread of disease, identify the potentially risk population exposed and creating measures for other strategic interventions such as welfare programmes or giving assistance or subsidies to really affected and high priority families.

Demarcating the vulnerable population or COVID 19 clusters to some geographical extent is a challenge. Grama Niladhari Division (GND) is the smallest administrative boundary of Sri Lanka and demarcation is clearly defined and digitized the maps for GIS purposes by the Department of Survey General. These GND information is used to demarcate the COVID 19 risk area supported with other COVID 19 related epidemiological risk parameters.

\section{Criteria to categorise High Risk GND}

1) There are the positive cases reported beyond particular family cluster

2) There are positive secondary contacts

3) There are positive cases without epidemiological link (Unknown Index Case)

4) There are unknown contacts in the area

5) Positive cases reported after participation to an event (Religious, Wedding or Party)

6) PCR positivity rate

Freely available GND information (Digital Data, shape files) from Department of Survey General is used as based maps. MOH Area Map layers and District Map layers were created using same GND data source in same resolution. QGIS vision3.16, MS Excel and Google Sheet were used as computer software. 
Each and every COVID 19 positive case reported at high risk $\mathrm{MOH}$ areas was entered to the Google Sheet and updated all relevant epidemiological information. Geographical coordinates were captured for each and every case and was updated using centroid coordinates of relevant GND. This information was used later with QGIS software to create Risk and Other COVID 19 Map production. Random spotting of cases by GND was the major algorithm used to maintain privacy and confidentially of the patients.

\section{Results and Discussion}

\section{Use of GIS in Management of COVID 19 in PDHS, NWP}

GIS mapping was broadly used by the Provincial Department of Health Services, North Western Province (PDHS NWP) for management of COVID 19in two situations;

i. For clear demarcation of lock down areas until contact tracing

ii. Socio economic stratification of lock down areas for resource allocation.

\section{Clear demarcation of lock down areas until contact tracing}

The contact tracing of COVID 19 patients remains a priority in controlling spurious spread $(9,10)$. However the contact tracing in the developing countries is somewhat hard due to lack of systematic location tracing methods(11).

On 12/10/2020, a COVID 19 patient was detected by the passive surveillance system established at the Teaching Hospital Kuliyapitiya. This was a random PCR testing conducted for febrile patients presenting to outpatient department. A complex situation developed while tracing her contacts. She has participated to a wedding and a mass at the church. All her workplace colleagues were tested negative. On 15/10/2020, two of the participants at the wedding were tested positive. On 16/10/2020, a patient who was admitted for heart failure was tested positive for COVID 19, exposing
53 members of the hospital mass. This incident and the untraceable numerous contacts lead to a situation out of control from the health officials.

Locking down the whole region was going to affect the country as most of the egg and other dairy product needs are supplied from this area. All the cases and known contacts of this incident that has been found were mapped using GIS mapping. Four Grama Niladhari Divisions were found to be red spots. GIS map was used to prioritise the PCR testing of the contacts and community. As all the cases were mapped separately, it was easy to identify the affected GN divisions of this immediate incident, separately. Curfew was imposed in these GN areas without affecting the other areas of the $\mathrm{MOH}$.

\section{Figure 1- COVID 19 cases distribution in Kuliyapitiya $\mathrm{MOH}$ area}


As shown in the figure 1, the cases marked by fire flame were the cases related to the incident mentioned above. The areas demarcated with these were locked down immediately and an urgent contact tracing was conducted. This map enabled as to lockdown targeted areas with high disease burden.

\section{Socio economic stratification of lock down areas for resource allocation}

Lockdown was a strategy used in COVID 19 outbreak in many countries $(12,13)$. This is necessary in contact tracing and preventing further spread of disease. But some of the residents in these areas are daily wagers with low income levels. Lock down will push them below poverty line more and requiring provision of daily needs (14). Therefore, government has decided to provide basic needs and some monetary allowances for people in these areas. For a developing country, such decision should be initiated among most needy people as of the poor economic strength in the country. Therefore, socio economic data should be apparent for such distribution. This has been conducted in USA with use of mapping of socio economic status and COVID 19 cases for the purpose of disease exploration (15). At PDHS NWP this method was crudely used in outbreaks at Kurunegala municipal council areas. Some areas in Municipal council area were locked down with identification of several sub clusters. After mapping, it was found that these sub clusters were spread throughout the city limits. A local government decision was taken to provide basic needs and monetary allowance for the people. In there the prioritisation had to be done using the socio economic stratification. GIS mapping was conducted stratifying areas according to Socio economic data available. This was further furnished with COVID 19 case distribution mapping. The clusters in lowest socio economic levels were identified and prioritised in aid distribution.

\section{Figure 2COVID 19 Local Level Sub Cluster for Essential Lockdown}
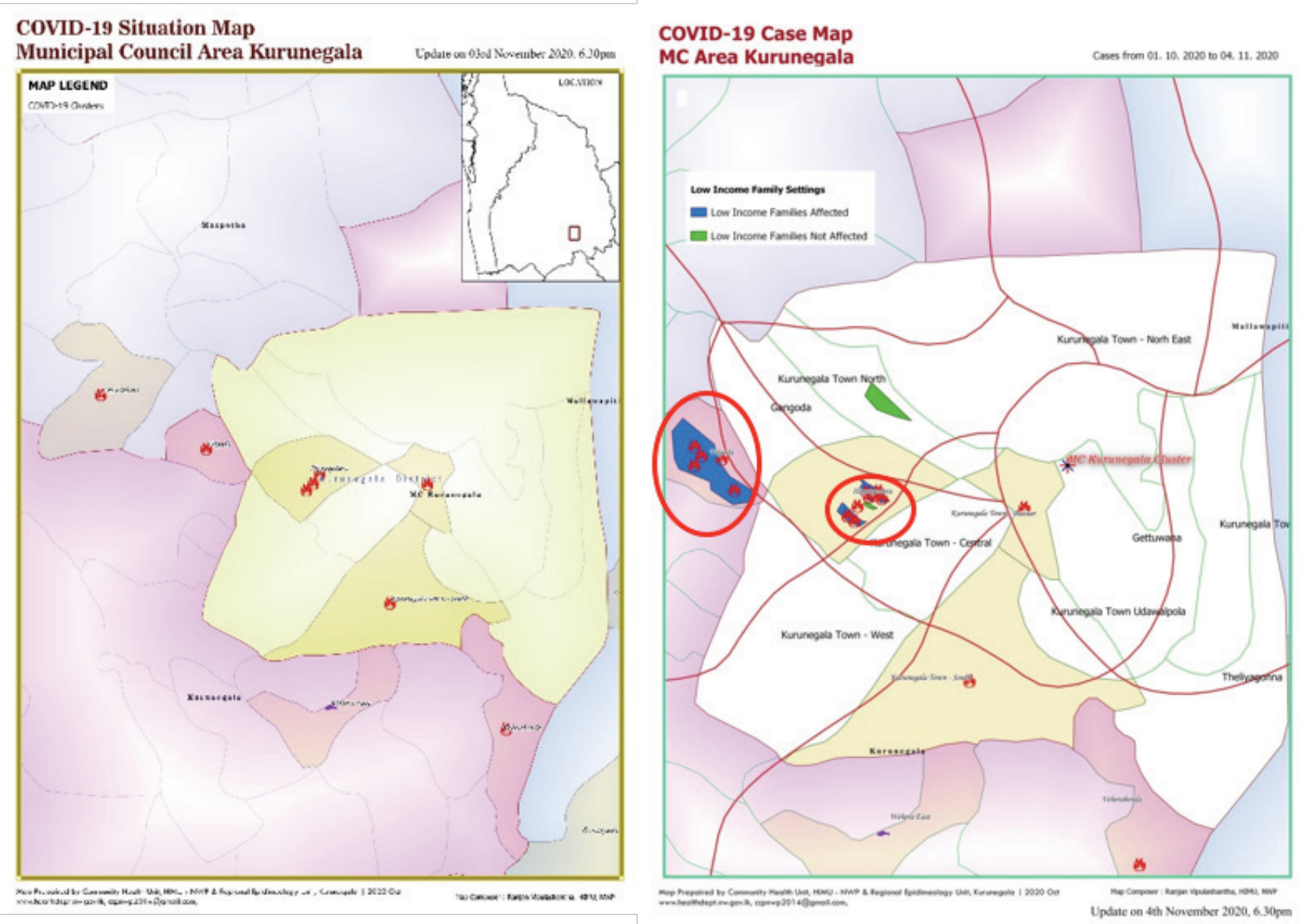


\section{Conclusion}

GIS can be incorporated in COVID-19 outbreak management process in different scenarios at regional level. Intersectoral information sharing could be achieved by utilizing GIS Maps in a user friendly way. Further high risk geographic areas can be visualized and demarcated at GND level as well as below GND levels. Decision of restriction of people movements or lock down can be taken cautiously by exploring GIS maps. In addition it improves transparency of data and case reporting. Ultimately it will facilitate evidence based sociological and epidemiological outbreak management decisions.

\section{Further development}

This GIS mapping can be incorporated in to a data base developed on COVID 19 patients. Thus automatically all the maps can be generated. It will facilitate automatic new cluster identification and contributions in COVID 19 distribution analysis.

\section{Reference}

1. Murugesan B, Karuppannan S, Mengistie AT, Ranganathan M, Gopalakrishnan G. Distribution and Trend Analysis of COVID-19 in India: Geospatial Approach. Journal of Geographical Studies. 2020;4(1):1-9.

2. Lakhani A. Which Melbourne metropolitan areas are vulnerable to COVID-19 based on age, disability, and access to health services? using spatial analysis to identify service gaps and inform delivery. Journal of pain and symptom management. 2020;60(1):e41-e4.

3. Gibson L, Rush D. Novel coronavirus in Cape Town informal settlements: feasibility of using informal dwelling outlines to identify high risk areas for COVID-19 transmission from a social distancing perspective. JMIR Public health and surveillance. 2020;6(2):e18844.

4. Adam M, Bärnighausen T, McMahon SA. Design for extreme scalability: A wordless, globally scalable COVID-19 prevention animation for rapid public health communication. Journal of Global Health. 2020;10(1).

5. WHO. Rolling updates on coronavirus disease (COVID-19) 2020 [cited 2020]. Available from: https://www.who.int/emergencies/diseases/novelcoronavirus-2019/events-as-they-happen.
6. Worldometer. COVID-19 CORONAVIRUS PANDEMIC 2021 [cited 2021]. Available from: https://www.worldometers.info/coronavirus/.

7. Wickramaarachchi W, Perera S, Jayasinghe S. COVID-19 epidemic in Sri Lanka: A mathematical and computational modelling approach to control. Computational and mathematical methods in medicine. 2020;2020.

8. PDHS-NWP. COVID-19 Weekly Reports. PDHSNWP WEBSITE: Provincial Department of Health Services-North Western Province, 2021.

9. Salathé M, Althaus CL, Neher R, Stringhini S, Hodcroft E, Fellay J, et al. COVID-19 epidemic in Switzerland: on the importance of testing, contact tracing and isolation. Swiss medical weekly. 2020;150(1112).

10 Cheng H-Y, Jian S-W, Liu D-P, Ng T-C, Huang $\mathrm{W}-\mathrm{T}$, Lin H-H. Contact tracing assessment of COVID-19 transmission dynamics in Taiwan and risk at different exposure periods before and after symptom onset. JAMA internal medicine. 2020;180(9):1156-63.

11. Mbunge E. Integrating emerging technologies into COVID-19 contact tracing: Opportunities, challenges and pitfalls. Diabetes \& Metabolic Syndrome: Clinical Research \& Reviews. 2020;14(6):1631-6.

12. Melnick ER, Ioannidis JP. Should governments continue lockdown to slow the spread of covid-19? BMJ. 2020;369.

13. Di Domenico L, Pullano G, Sabbatini CE, Boëlle P-Y, Colizza V. Impact of lockdown on COVID-19 epidemic in Île-de-France and possible exit strategies. BMC medicine. 2020;18(1):1-13.

14. Anser MK, Yousaf Z, Khan MA, Nassani AA, Alotaibi SM, Abro MMQ, et al. Does communicable diseases (including COVID-19) may increase global poverty risk? A cloud on the horizon. Environmental Research. 2020;187:109668.

15. Mollalo A, Vahedi B, Rivera KM. GIS-based spatial modeling of COVID-19 incidence rate in the continental United States. Science of the total environment. 2020;728:138884. 